\title{
A role of stimulus compounds in eliciting responses: Relatively spaced extinction following massed acquisition
}

\author{
E. J. CAPALDI and MICHAEL D. MORRIS \\ Purdue University, West Lafayette, Indiana 47907
}

\begin{abstract}
One of two schedules of rewarded $(R)$ and nonrewarded (N) trials (RNR vs. RRN) was combined factorially with intertrial interval (ITI) in acquisition (1 vs. $45 \mathrm{~min}$ ), with extinction occurring at a 45-min ITI. The RNR schedule produced greater resistance to extinction than the RRN schedule regardless of acquisition ITI. The shift in ITI from acquisition to extinction reduced resistance to extinction slightly in the RNR group but not in the RRN group. These findings suggest that there are cues common to 1 -min and $45-\mathrm{min}$ ITIs. It was suggested that these ITI-associated cues enter into compound with memories to control instrumental responding.
\end{abstract}

A change in intertrial interval (ITI) from acquisition to extinction reduces resistance to extinction and reduces or eliminates reward-schedule effects such as the partial reinforcement extinction effect (Amsel, Wong. \& Traupmann, 1971: Capaldi. Berg, \& Sparling, 1971: Capaldi \& Morris, 1974; Lew1s \& Cotton, 1959; Teichner, 1952). Collectively, these findings indicate (see Capald \& \& Morris, 1974) that massed trial responding is controlled by stimulus traces (STrs) produced by reward and nonreward, that not only are unique stimuli associated with highly massed trials (the STrs) but with Trial 1 of the day (ST1) as well, and that memories such as the memory of nonreward $\left(\mathrm{S}^{\mathrm{N}}\right)$ enter into compound with $\mathrm{STr}$ and ST1 to regulate instrumental responding.

According to Capaldi and Morris (1974), if, as under massed trial partial reinforcement, the stimulus compound $\mathrm{S}^{\mathrm{N}}+\mathrm{STr}$ acquires associative control over the instrumental reaction, then the stimulus compound $\mathrm{SN}^{\mathrm{N}}+\mathrm{ST} 1$ which occurs in extinction at a $24-\mathrm{h}$ ITI will possess but little capacity to elicit the instrumental reaction, and vice versa. The role of the stimulus compounds in eliciting responses cannot be stressed too greatly; it is the compounds and not $\mathrm{S}^{\mathrm{N}}$ alone which elic1t responses in acquisition and extinction. Moreover, ITI-associated cues such as STr and ST1 are retrieval cues for memories such as $\mathrm{S}^{\mathrm{N}}$, and thus these cues determine, in combination with other retrieval cues such as alley cues, on what particular trials $\mathrm{S}^{\mathrm{N}}$ will be present, i.e., retrieved. For example, if nonreward occurs on Trial 1 of the day, i.e., $\mathrm{S}^{N}$ is stored in the presence of ST1, then $S^{N}$ will be better retrieved on Trial 1 of the following day, when ST1 is presented again, than on Trial 2 of the same day, where ST 1 is absent, and STrs are present (see Capaldi \& Morris, 1974). That SN stored on Trial 1 may be better retrieved on Trial 1 of the following day than on Trial 2 of the same day is

This research was supported in part by National Institute of Child Health and Development Grant 5 Ro1 HD04379-04 to E.
J. Capaldi. perhaps counterintuitive, but it is consistent with recent findings (Capaldi \& Morris, 1974) as well as with the view that not the passage of time per se, but the absence of retrieval cues, is the basis of forgetting (e.g.. Capaldi, 1971; Tulving, 1974).

Granting that memories such as $\mathrm{S}^{\mathrm{N}}$ always enter into compound with ITI-associated cues such as STr and ST1 to control responding, and that STr and ST1 act as retrieval cues for $\mathrm{S}^{\mathrm{N}}$, it follows that in order to understand responding at a particular ITI, it is necessary to determine if unique cues are associated with that ITI. However, since shifts in ITI from acquisition to extinction have only occurred from or to a 24-h ITI (e.g.. Amsel et al., 1971; Capaldi et al., 1971; Mackintosh \& Little, 1970) or from one to another relatively short ITI (e.g., Lewis \& Cotton, 1959; Teichner, 1952), information is lacking as to what unique cues, if any, are associated with trials sufficiently spaced so that STrs are not present, but neither are the ST 1 cues, since the ITI is less than $24 \mathrm{~h}$. This matter was of concern in the present investigation, in which rats given acquisition training at a 1-min ITI were subsequently extinguished at a 45 -min ITI.

In this investigation, groups received a schedule of rewarded $(\mathrm{R})$ and nonrewarded $(\mathrm{N})$ trials which either contained (Group RNR) or did not contain (Group RRN) a daily transition from $\mathrm{N}$ to $\mathrm{R}$ trials. Two findings concerning the effects of these schedules on extinction are noteworthy here; if training and extinction occur at a relatively short ITI, RNR groups show much greater resistance to extinction than RRN groups (e.g., Capaldi \& Lynch, 1968), but if training occurs at a short ITI followed by extinction at a $24-\mathrm{h}$ ITI, the RNR schedule will produce no greater resistance to extinction than the RRN schedule (Mackintosh \& Little, 1970). Of concern here were the effects of extinguishing RNR and RRN groups at a 45-min ITI following training at a $1-\mathrm{min}$ ITI. Also included in this investigation were RNR and RRN groups trained and extinguished at a 45-min ITI. 


\section{METHOD}

\section{Subjects}

The 40 male albıno rats, approximately 130 days old at the beginning of the experiment, were purchased from Holtzman Co., Madison. Wisconsin.

\section{Apparatus}

The apparatus was a runway $208.4 \mathrm{~cm}$ long $\times 22.0 \mathrm{~cm}$ high and $10.2 \mathrm{~cm}$ wide with a hinged lid of $1.3 \mathrm{-cm}$ hardware cloth and a wood floor. The alley was divided into three sections The first section consisted of a treadle $20.3 \mathrm{~cm}$ long, suspended above a microswitch. By placing the front paws of the rat on the forward edge of the treadle, the first clock $(.01 \mathrm{sec})$ was started. Interruption of a photobeam $5.1 \mathrm{~cm}$ beyond the treadle stopped Clock 1 (start time) and activated Clock 2 (run time). Interruption of a photobeam $132.1 \mathrm{~cm}$ beyond the first photobeam stopped Clock 2 and activated Clock 3 (goal tıme). The goal clock was stopped when a photobeam $39.4 \mathrm{~cm}$ beyond the second photobeam and $6.4 \mathrm{~cm}$ in front of a $5.1 \times 10.2 \times 3.8 \mathrm{~cm}$ food tray was broken. Within the food tray was a $3.8 \times 3.2 \times 1.9 \mathrm{~cm}$ metal foodcup which was positioned on the right side of the food tray. A brass gullotine door, $30.5 \mathrm{~cm}$ from the end of the runway, was manually lowered to prevent retracing once an animal had interrupted the third photobeam.

\section{Procedure}

Upon arrival at the laboratory, the subjects were mantaned on ad ad-lib diet for approximately 40 days. Water was always avalable in the home cages. On Day 1 of the experiment, all food was removed from the subjects' cages Throughout the remainder of the experiment, subjects were maintained on a 13-g/day deprivation schedule. On Day 10 and each of the 2 following days, subjects were handled in squads of four for $4 \mathrm{~mm} / \mathrm{squad}$. After the daly handling session, subjects were returned to the home cages where they received a glass caster containing 15 .045 -g Noyes pellets On Day 13, subjects were randomly assigned to one of four treatment groups defined by the factorial combination of acquisition ITI and acquisition sequence. The two acquisition ITIs were $1 \mathrm{~min}$ and $45 \mathrm{~mm}$, whle the sequences were defined by whether $R$ trials followed $N$ trials within a day (RNR condition) or by whether all N trials followed all daily $\mathrm{R}$ trals (RRN condition) Ten subjects were assigned to each of the

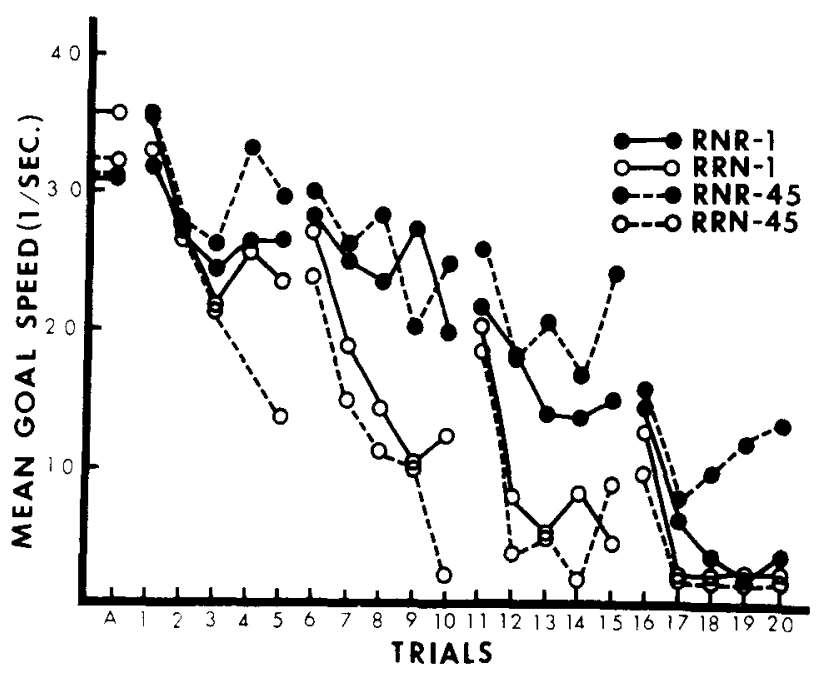

Figure 1. Mean goal speed for the four experim ental groups for the last day of acquisition and each trial of the 4 days of extinction. four treatment groups The 1-min ITI groups (Group RNR-1 and Group RRN-1) were run in squads of two. one from each sequence condition The running order within each squad was randomly determined each day. The subjects in a squad were then run alternately untal all trials of a day were completed. This squad rotation produced an ITI of about 1 min. Running order between squads was the same each acquisition day. The 45-min ITI groups (RNR-45 and RRN-45) were rotated such that all 20 subjects in this condition finished a trial before the rotation was begun again Each acquisition day. subjects were randomly assigned a running order within squads of two, one subject representing each sequence condition such that the subjects in Squad 1 were assigned Running Orders 1 and ?, and subjects in Squad 2 were assigned Running Orders 3 and 4 , and so forth, until all 20 subjects were assigned an order. This rotation would normally produce an ITI of less than $45 \mathrm{~min}$, so the experimenter did not begin the next rotation until 45 min had elapsed.

The two acquisition sequences were administered for 10 days. The subjects in the RNR condition received the following order of $\mathrm{R}$ and $\mathrm{N}$ trials (where a comma separates days): RNR, RNNRR, RRNR, RNR, RNNR, RRNR, RNR, RRNR, RNR, and RRNR, while subjects in the RRN condition received RRN, RRRNN, RRRN, RRN, RRNN, RRRN, RRN, RRRN, RRN, and RRRN. It should be noted that these two sequence conditions are equated for number of trials, number of $R$ trials, and number of $\mathrm{N}$ trials on both a dally and an overall basis. The only difference between the conditions is the sequence of $R$ and $\mathrm{N}$ trals. On all $\mathrm{R}$ trials, subjects were administered 15 .045-g Noyes pellets and remained in the goalbox until all pellets had been . consumed. On $\wedge$ trals, subjects were confined in the goalbox for $30 \mathrm{sec}$

Beginning the dav after the last day of acquisition training. extinction was administered at a rate of five trials per day for 4 days. Each squad of two subjects in acqusition was combined with the same numbered squad from the other ITI condition. Dally running orders were randomly assigned within these new squads of four subjects such that the subjects in Squad 1 were assigned Orders 14 , the subjects in Squad 2 were assigned Orders 5-8, and so forth All 40 subjects received a trial in rotation prior to the next rotation. The ITI was not allowed to fall below $45 \mathrm{~mm}$. Nonreward confinement duration remained at $30 \mathrm{sec}$

\section{RESULTS}

The times recorded from each alley section were converted to speeds $(1 / \mathrm{sec})$ and all analyses were performed on these speeds. Since the run and goal sections produced similar results and the start section revealed no significant differences attributable to the experimental variables, only the goal-section findings will be presented here.

On the last day of acquisition, Group RRN-1 appeared to be running faster than the otler three groups. Analysis of variance revealed, however, that sequence $[F(1,36)=2.25, \mathrm{p}>.10]$, IT $](F<1.0)$, and the Sequence by ITI interaction were not significant $(\mathrm{F}<1)$.

The mean goal speed on the last day of acquisition and the goal speeds for each trial of extinction for the four experimental conditions are shown in Figure 1. Examination of Figure 1 reveals that the RNR groups ran faster in extinction than the RRN groups. Too, the differences between the 1-min acqusition ITI groups was smaller on Days 1 and 4 than the differences between 
the 45-min acquisition ITI groups. While the smaller difference on Day 1 may be attributed to terminal acquisition differences between Groups RNR-1 and RRN-1, it is clear that reduced differences on Day 4 are due to the slow responding of Group RNR-1 as compared to Group RNR-45.

In an earlier or "pilot" version of this investigation, extinction differences between Groups RNR-1 and RNR-45 developed as shown in Figure 1; the two groups differed little on early extinction days and then, as on Day 4 in Figure 1, Group RNR-1 ran very slowly relative to Group RNR-45. Given these preliminary findings, it was decided to statistically analyze each day of extinction separately.

A 2 (sequences) by 2 (ITIs) by 4 (trials/day) analysis of variance was performed on each extinction day. Trial 1 of each day was excluded since it did not reflect performance under a 45 -min ITI. That the RNR groups were more resistant to extinction than the RRN groups was revealed by significant sequence effects on Day 2 $[F(1,36)=19.15, p<.001]$, Day $3 \quad[F(1,36)=13.72$, $p<.005]$ and Day $4[F(1,36)=9.67, p<.005]$. On Day 1 , the sequence effect was marginally reliable $[F(1,36)=3.88, p<.06]$. Individual comparisons of the effect of sequence at each ITI revealed that Group RNR-45 ran faster than Group RRN-45 on all 4 extinction days [smallest $\mathrm{F}(1,36)=5.85, \mathrm{p}<.05$ ] while Group RNR-1 was significantly faster than Group RRN-1 on Day $2[F(1,36)=6.49, p<.05]$ and Day $3[\mathrm{~F}(1,36)=8.11, \mathrm{p}<.005]$ but not on Days 1 and $4(\mathrm{Fs}<1)$. That the $\mathrm{N}-\mathrm{R}$ sequence effect was smaller for the 1-min ITI groups than for the 45-min ITI groups was indicated by a significant Sequence by ITI interaction on Day 4 of extinction $[F(1,36)=4.96, p<.05]$. Simple effects of this interaction further indicated that Group RNR-1 was significantly less resistant to extinction than was Group RNR-45 $[\mathrm{F}(1,36)=7.78$, $p<.01]$ while the RRN groups did not differ $(F<1)$.

\section{DISCUSSION}

The present findings that Group RNR-1 showed a slight, but not a substantial, decrease in resistance to extinction, and that Group RNR-1 was more resistant to extinction than was Group RRN-1 suggests that a 1-min ITI and a 45-min ITI are not entirely dissimilar, and that the two ITIs share cues in common. In keeping with prior usage (Capaldi \& Morris, 1974), the cues common to ITIs diverse as $1 \mathrm{~min}$ and $45 \mathrm{~min}$ are called cues common to massed trials and are symbolized as SMT. While the basis of the SMT cues is at this time a matter of conjecture, it is possible to identify events which may not lie at the basis of the SMT cues. The SMT cues would not seem to be STrs produced by reward and nonreward, for by common agreement such STrs are not considered to remain active for so long as 45 min (e.g., Amsel, 1967; Hull, 1952; Rothkopf, 1955; Sheffield, 1949; Teichner, 1952). Of course, if subsequent data indicated the STrs produced by reward and nonreward do remain active for as long as $45 \mathrm{~min}$, then the hypothesis that STrs are at the basis of the SMT cues would be tenable. We shall, however, treat STrs and SMT cues separately here. In any event, in the Mackintosh and Little (1970) investigation, RNR and RRN groups trained at a 30 -sec ITI failed to differ in spaced $(24 \mathrm{~h})$ extinction. Thus, the SMT cues must be reasonably dissimilar from the ST1 cues, for if this were not so, then Groups RNR-1 and RRN-1 of this investigation would have failed to differ in extinction at a 45-min ITI as did the RNR and RRN groups of Mackintosh and Little (1970) shifted to a 24-h extinction ITI. On the basis of available ITI-shift findings, then, three sorts of ITI-associated cues may be identified, STrs produced by reward and nonreward which are present only at highly massed trials, ST1 cues which are present only on the first trial of the day, and SMT cues which appear to be present on all trials of the day except Trial 1 and which may be relatively independent of within-day ITI. Employing these cues, let us apply the memory model (e.g., Capaldi \& Morris, 1974) to the different results obtained by Mackintosh and Little (1970) and in the present investigation.

The tendency to resist extinction at a $24 \cdot \mathrm{h} \mathrm{ITI}$ depends upon the capacity of the stimulus compound $\mathrm{S}^{N}+\mathrm{ST} 1$ to elicit the seaction. In neither Group RNK nor Group RRN employed by Mackintosh and Little (1970) did the stimulus compound SN + ST1 acquire in acquisition any appreciable tendency to elicit the instrumental reaction. The within-day ITI in the Mackintosh and Little investigation was $30 \mathrm{sec}$. Thus, in both Group RNR and Group RRN, $\mathrm{S}^{\mathrm{N}}$ was stored in the presence of STr + SMT and thus in neither group would $\mathrm{S}^{N}$ be appreciably retrieved by ST1 or on the first trial of the subsequent day. In neither group, then, did $\mathrm{S}^{\mathbf{N}}$ occur strongly in compound with ST1 in acquisition, and thus the introduction of the $\mathrm{S}^{\mathrm{N}}+\mathrm{ST} 1$ stimulus compound in extinction failed to elicit the reaction with any degree of vigor in either of the groups. In an investigation by Capaldi and Lynch (1968), an RNR group was more resistant to extinction than an RRN group when training and extinction occurred at a 3-min ITI. Assuming that STrs are present at a 3-min ITI, the tendency to resist extinction at that ITI would depend upon the capacity of the stimulus compound $\mathrm{S}^{\mathrm{N}}+\mathrm{STr}+\mathrm{SMT}$ to elicit the reaction. In Group RNR $\mathrm{S}^{N}$ was stored in the presence of STr and SMT on Trial 2 of the day, and this stimulus compound was conditioned to the instrumental reaction on Trial 3 of acquisition days. However, there is no acquisition trial for Group RRN on which the stimulus compound $\mathrm{S}^{\mathrm{N}}+\mathrm{STr}+\mathrm{SMT}$ can occur and be conditioned to the reaction. Thus, Group RNR showed greater resistance to extinction than did Group RRN at a 3-min ITI.

The tendency to resist extinction at a $45-\mathrm{min}$ ITI depends upon the capacity of the stimulus compound $\mathrm{S}^{\mathrm{N}}+\mathrm{SMT}$ to elicit the reaction. This stimulus 
compound was conditioned to the instrumental reaction in Group RNR-45 on rewarded trials which followed nonrewarded trials, but the compound $\mathrm{S}^{\mathrm{N}}+\mathrm{SMT}$ was not conditioned to the instrumental reaction in acquisition in Group RRN-45, because there was no acquisition trial on which this compound occurred for for Group RRN. Accordingly, Group RNR45 showed greater resistance to extinction than did Group RRN-45. In Group RNR-1, the stimulus compound conditioned to the instrumental reaction in acquisition on rewarded trials which followed nonrewarded trials was $\mathrm{SN}^{\mathrm{N}}+\mathrm{STr}+\mathrm{SMT}$; this compound was not conditioned to the instrumental reaction in Group RRN-i, because on no tiial did this compounu occur in Group RRN.1 in acquisition. In order to understand the considerable resistance to extunction of Group RNR-1, then, it need only be assumed that if the stimulus compound $\mathrm{SN}^{\mathrm{N}}+\mathrm{STr}+\mathrm{SMT}$ is conditioned to the instrumental reaction, then the compound $\mathrm{S}^{N}+\mathrm{SMT}$ will elicit the instrumental reaction with considerable vigor. That Group RNR-1 was slightly less resistant to extinction than was Group RNR-45 was presumably due to the fact that, whereas Group RNR 45 was extinguished in the presence of the stimulus compound which had directly acquired control over the instrumental reaction in acquisition $\left(S^{N}+S M T\right)$, in Group RNR-1 the extinction compound ( $\mathrm{S}^{\mathrm{N}}+\mathrm{SMT}$ ) differed slightly from the compound conditioned to the response in acquisition $\left(S^{N}+S T r+S M T\right)$. The effects in extinction in Group RNR-1 of removing STr from the stimulus compound were quite evidently slight, inasmuch as Group RNR-1 was not much less resistant to extinction than was Group RNR-45. The effect is much greater, of course, when, as in the Mackintosh and Little investigation, an RNR group was extinguished at a 24-h ITI, for at a 24-h extunction ITI, not only are both STr and SMT removed from the stimulus compound but ST1 is introduced in compound along with $\mathrm{S}^{\mathrm{N}}$, as well. The present model is consistent with all extinction findings which have been reported for RNR vs RRN groups. Moreover, it is consistent with other ITI-shift findings which have been reported (see Capaldi \& Morris, 1974).

In many instances, neither ITI nor apparatus cues nor other cues change meaningfully from one trial to the next, and in such cases it is irrelevant to the predictions of the model employed whether or not the stimulus compounds which control behavior are emphasized. Thus, for example, the predictions of the memory model are not altered for, e.g., a partially rewarded group which is trained and extinguished at a 24-h ITI no matter if the compound $\mathrm{S}^{\mathrm{N}}+\mathrm{ST} 1$ is emphasized or merely $S^{N}$ is emphasized. However. when, as in the present investigation. ITI is changed from acquisition to extinction. it becomes necessary to specify precisely and in detail the total stimulus compound which controls responding. Accordingly, one of the major contributions of ITl-shift findungs to contemporary theory may lie in forcefully bringing to attention that memories such as SN always enter into compound with whatever other cues may be avalable to control instrumental responding. For example, on the basis of within-subjects partıal-renforcement findings (see Capaldi, 1971; Capaldi \& Morr1s, 1974, Mellgren \& Dyck, 1972; Mellgren, Dyck. Seybert, \& Wrather, 1973), it is clear that in discrimination learning situations $S^{N}$ enters into compound with visual cues. e.g., black alley vis. white alley, to control instrumental responding. Indeed, Burstein and Moeser (1971) have demonstrated that brightness cues enter into compound with ST1 cues to reguiate instrumentai behavior. Thus, that stimulus compounds are not always emphasized, as here, means only that in many cases such specification is not necessary. Clearly. however, such specification is important, for a model which can explain, e.g., a partial reinforcement extinction effect when the ITI is the same in extinction as in acquisition may not be able to explain the effects of an ITI shift on the partial reinforcement extinction effect. Generally speaking, then, ITI-shift findings appear to demand a more precise specification of the stimuli controlling behavior.

\section{REFERENCES}

Amsel, A. Partial reinforcement effect on vigor and persistence: Advances in frustration theory derived from a variety of within subject experiments. In K. W. Spence and J. T. Spence (Eds.), The psichology of learning and motivation Advances in research and theory Vol. 1 New York: Academic Press, 1967.

Amsel, A., Wong, T. P., \& Traupmann, K, L. Short-term and long-term factors in extinction and durable persistence Journal of Experimental Psychology, 1971, 90,90-95.

Burstein, K. R., \& Moeser, S. The informational value of a distinctive stimulus associated with the initiation of acquisition trials. Learning and Motivation, 1971, 2, 228-234.

Capaldı, E. J. Partial reinforcement: A hypothesis of sequential effects. Psychological Review, $1966,73,459-477$.

Capaldı, E J. Memory and learning: A sequential vewpoint. In W. K. Honig and P. H. R. James (Eds.), Anımal memory'. New York: Academic Press, 1971.

Capaldı, E. J., Berg, R. F., \& Sparling, D. L. Trial spacing and emotionality in the rat. Journal of Comparative and Physiological Psychology, 1971, 76, 290-299.

Capaldi, E. J., \& Lynch, A. D. Magnitude of partial reward and resistance to extinction: Effect of N-R transitions. Journal of Comparative and Physiological Psychology, 1968, 65, $179-181$.

Capaldi, E. J., \& Morns, M. D. Reward schedule effects in extinction: Intertrial interval, memory and memory retrieval. Learning and Motivation, 1974, 5, 473-483.

Hull, C. L. A behalior system New Haven: Yale University Press, 1952 .

Lewis, D. J., \& Cotton, J. W. The effect of intertrial interval and number of acquisition trials with partial reinforcement or performance. Journal of Comparative and Physiological Psychology, 1959, 52, 398-601.

Mackintosh, N. J., \& Little, L. Effects of different patterns of reinforcement on performance under massed and spaced extinction. Psvchonomic Science. 1970, 20, 1-2.

Mellgren, R. L., \& Dyck, D. G. Partial reinforcement effect, reverse partial reinforcement effect, and generalized partial reinforcement effect within subjects. Journal of Expenmental reinforcement effect within subj
Psychology, 1972, 92, 330-346.

Mellgren, R. L., Dyck, D. G., Seybert, J. A., \& Wrather, D. M. Within subjects partial reinforcement effects: Reward-nonreward transitions and generalization. Journal of Experimental Psychology, 1973, 99, 389-394.

Rothkopf, E. Z. Distribution of practice and temporal decay of response proauced stimul. Journal of Experimental Psychology, 1955. 49, 33-38. 
Sheffield, V. F. Extinction as a function of partial reinforcement and distribution of practice. Journal of Experimental Psychology, 1949, 39, 511-526.

Teichner, W. H. Experimental extinction as a function of the intertrial intervals dunng conditioning and extinction. Journal of Experimental Psychology, 1952, 44, 170-178.
Tulving, E. Cue-dependent forgetting. American Scientist, 1974, $62,74-82$.

(Received for publication May 20, 1974: revision recelved June 28,1974 .) 